\title{
Enfermería de urgencias en la intubación de secuencia rápida a pacientes COVID-19
}

Emergency Nursing Care for Rapid Sequence Intubation in COVID-19 Patients

Cuidados de enfermagem de emergência para intubação de sequência rápida em pacientes com COVID-19

Como citar este art culo:

Acevedo Gamboa Fanny Esperanza, Páez Barrozo Mansur, Mayorga Camargo Viviana Lizeth. Enfermería de urgencias en la intubación de secuencia rápida a pacientes COVID-19. Revista Cuidarte. 2020;11(3):e1319. http://dx.doi.org/10.15649/ cuidarte.1319

Revista Cuidarte

Rev Cuid. 2020; 11(3): e1319

doij $h$ ttp://dx.doi.org/10.15649/cuidarte.1319

E-ISSN: 2346-3414

(1) Fanny Esperanza Acevedo Gamboa'

(1) Mansur Páez Barrozo²

(1) Viviana Lizeth Mayorga Camargo ${ }^{3}$

1 Profesora Departamento de Enfermería Clínica. Urgencias. Pontificia Universidad Javeriana. Bogotá, Colombia. facevedo@javeriana.edu.co Autor de correspondencia

2 Enfermero área de urgencias. Fundación Santafé de Bogotá. Bogotá, Colombia. mansur.paez@fsfb.org.co

3 Enfermera área de urgencias. Hospital Universitario San Ignacio. Bogotá, Colombia. vlmayorga@husi.org.co

\section{Resumen}

Introducción: La inducción de secuencia rápida modificada es la sugerida para la intubación orotraqueal a pacientes COVID-19 que presentan distrés respiratorio en urgencias. El objetivo de esta revisión es plantear el proceso de atención de enfermería durante las fases de planificación, preparación, pretratamiento y sedación con parálisis neuromuscular. Materiales y Métodos: artículo de reflexión acerca de elementos conceptuales en la valoración, diagnóstico, planeación, ejecución y evaluación del cuidado de enfermería, a partir de la revisión de literatura de consensos y protocolos nacionales e internacionales y publicaciones de cuidado de enfermería a pacientes COVID-19. Resultados: Ios medicamentos recomendados por la literatura en la inducción de secuencia rápida a pacientes COVID-19 son: en la fase de pretratamiento, las benzodiacepinas necesarias para disminuir la ansiedad del paciente, la lidocaína que controla el reflejo de la tos y el fentanilo que contribuye con la analgesia; en la fase de sedación inductores como etomidato y ketamina están indicados en pacientes con inestabilidad hemodinámica y propofol en pacientes estables; en la fase de parálisis neuromuscular el rocuronio es el recomendado como primera opción antes que la succinilcolina y los vasopresores tipo norepinefrina, epinefrina, vasopresina y/o dopamina, son necesarios en todas las fases para el manejo de la inestabilidad hemodinámica. Conclusiones: aplicar el proceso de atención de enfermería durante las fases de inducción de secuencia rápida contribuye con la organización de los medicamentos requeridos para la intubación orotraqueal, lo cual, disminuye la ansiedad del paciente y el riesgo de contaminación del personal de salud.

Palabras clave: Intubación e Inducción de Secuencia Rápida; Enfermería; Cuidados Críticos; Infecciones por Coronavirus; Urgencias Médicas.

Recibido: julio 17 de 2019

Aceptado: agosto 3 de 2020

Publicado: septiembre 3 de 2020 $\square *$ Correspondence

Fanny Esperanza Acevedo Gamboa

E-mail:facevedo@javeriana.edu.co 


\section{Emergency Nursing Care for Rapid Sequence Intubation in COVID-19 Patients}

\section{Abstract}

Introduction: Modified rapid sequence induction is suggested for orotracheal intubation in COVID-19 patients with respiratory distress in the emergency department. The objective of this review is to discuss nursing care during the phases of planning, preparation, pretreatment and sedation with neuromuscular paralysis. Materials and Methods: A reflection article was written on conceptual elements in nursing care assessment, diagnosis, planning, implementation and evaluation based on the literature review of national and international standards and protocols, and publications on nursing care in COVID-19 patients. Results: Drugs recommended by rapid sequence intubation in COVID-19 patients are benzodiazepine for reducing patient's anxiety, lidocaine for cough control and fentanyl for analgesia in the pretreatment phase; inducers such as etomidate and ketamine are suggested in hemodynamic unstable patients and propofol in stable patients in the sedation phase; rocuronium is recommended as the first-choice neuromuscular blocking agent before vasopressors such as succinylcholine and norepinephrine, epinephrine, vasopressin and/or dopamine which are needed in all phases for managing hemodynamic instability. Conclusions: Applying a nursing care plan during rapid sequence induction phases contributes to a more organized medication regimen required for orotracheal intubation, which reduces patient's anxiety and the risk of contamination for healthcare personnel.

Key words: Rapid Sequence Intubation and Induction; Nursing; Critical Care; Coronavirus Infections; Medical Emergencies

\section{Cuidados de enfermagem de emergência para intubação de sequência rápida em pacientes com COVID-19}

\section{Resumen}

Introdução: A indução de sequência rápida modificada é sugerida para intubação orotraqueal em pacientes com COVID-19 com problemas respiratórios no departamento de emergência. $\mathrm{O}$ objetivo desta revisão é discutir os cuidados de enfermagem durante as fases de planejamento, preparação, pré-tratamento e sedação com paralisia neuromuscular. Materiais e métodos: Um artigo de reflexão foi elaborado sobre elementos conceituais na avaliação, diagnóstico, planejamento, implementação e avaliação da cuidados de enfermagem com base na revisão da literatura de normas e protocolos nacionais e internacionais, e publicações sobre cuidados de enfermagem em pacientes com COVID-19. Resultados: As drogas recomendadas pela intubação de sequência rápida em pacientes com COVID-19 são benzodiazepinas para reduzir a ansiedade do paciente, lidocaína para controle da tosse e fentanil para analgesia na fase de pré-tratamento; indutores como etomidato e cetamina são sugeridos em pacientes com instabilidade hemodinâmica e propofol em pacientes estáveis na fase de sedação; o rocurônio é recomendado como o bloqueador neuromuscular de primeira opção frente a vasopressores como succinilcolina e norepinefrina, epinefrina, vasopressina e/ou dopamina, que são necessários em todas as fases para o manejo da instabilidade hemodinâmica. Conclusões: A aplicação de um plano de cuidados de enfermagem durante as fases de indução de sequência rápida contribui para a organização dos medicamentos necessários para a intubação orotraqueal, o que reduz a ansiedade do paciente e o risco de contaminação para o pessoal de saúde.

Palavras-chave: Intubação e Indução de Sequência Rápida; Enfermagem; Cuidados Críticos; Infecções por Coronavírus; Emergências Médicas. 


\section{Introducción}

La infección por SARS-CoV-2 que ocasiona la enfermedad de COVID-19, tiene como responsable a un nuevo virus de la familia Coronaviridae que compromete de manera especial el sistema respiratorio llevando al paciente a neumonía, edema pulmonar, síndrome de distrés respiratorio agudo (SDRA) o insuficiencia orgánica múltiple, sepsis y shock séptico ${ }^{1,2}$, reconociéndose la gravedad, cuando hay saturación de oxígeno menor a 90\%, frecuencia respiratoria mayor de 30 rpm y $\mathrm{PaO} 2 / \mathrm{FiO} 2 \leq 100 \mathrm{mmHg}^{3-5}$.

Desde el punto de vista fisiopatológico, el SDRA inicia con la entrada del virus por la vía respiratoria el cual llega al epitelio alveolar; la proteína $S$ del virus se une al receptor ECA II presente en los neumocitos II, los penetra y destruye, se activa la respuesta inflamatoria que libera citoquinas proinflamatorias en las células del endotelio y las células epiteliales, lo cual conduce a vasodilatación y aumento en la permeabilidad de la membrana alveolocapilar, ocasionando congestión del intersticio pulmonar y edema pulmonar difuso, se altera el intercambio gaseoso produciéndose hipoxemia severa, que sumado a la disminución de la distensibilidad y capacidad pulmonar residual como consecuencia de la lesión inflamatoria, obliga a que el paciente requiera de ventilación mecánica que permita mejorar el estado de oxigenación ${ }^{6-8}$.

La intubación orotraqueal para el inicio de ventilación mecánica en el paciente COVID-19 es un proceso de alto riesgo de contagio, por tanto, la Organización Mundial de la Salud $\mathrm{OMS}^{9}$ y la revisión de guías internacionales, sugiere la intubación e inducción de secuencia rápida (SIR) modificada que evite la ventilación manual del paciente lo que puede resultar en aerosolización del virus de las vías respiratorias ${ }^{10-13}$. “La intubación de secuencia rápida es apropiada después de una evaluación de la vía aérea que no identifica signos de intubación difícil"9.

El proceso de SIR, descrito por varios autores, lo componen 7 pasos: planificación y preparación el cual tiene que ver con la comprobación de todo el equipo necesario para llevar a cabo la intubación, preoxigenación que consiste en la aplicación de oxígeno durante 5 minutos con una mascarilla reservorio, pretratamiento que es la administración de fármacos antes de proceder a la inducción-relajación, sedación (inducción) con parálisis neuromuscular que consiste en llevar al paciente a la inconsciencia y relajación muscular, protección y posición del paciente es la colocación del paciente en una posición que optimice la visualización de la vía aérea, comprobación del tubo endotraqueal o dispositivo avanzado de la vía aérea es la verificación de la colocación del tubo mediante la visualización del tubo en las cuerdas vocales, auscultación y radiografía y manejo postintubación que comprende la sedación, analgesia y relajación con ajuste a los parámetros ventilatorios ${ }^{14-16}$.

La SIR modificada para pacientes COVID-19, recomienda aspectos importantes como lo son, el uso de un equipo de protección personal (EPP) especializado que debe utilizar el personal de salud que va a intervenir en la intubación orotraqueal ${ }^{17,18}$, preoxigenación con Fi02 del 100\% durante 5 minutos ${ }^{1,19}$, limitar las maniobras de preparación del paciente por riesgo de exposición del personal de salud, evitar maniobras de permeabilización de la vía aérea porque se consideran de alto riesgo de liberación de gotas o aerosoles que pueden contagiar al personal de salud, ventilación con máscara a dos manos con una gasa húmeda sobre la cara del paciente y debajo de la máscara para evitar la aerosolización, la no auscultación del tórax como estrategia de comprobación de la posición del tubo orotraqueal por el riesgo de contaminación del este- 
toscopio, para lo cual, se recomienda observar la expansión torácica bilateral simétrica con la ventilación, uso de video laringoscopio si se encuentra disponible para disminuir el contacto con la vía aérea y no ventilar hasta cuando el balón del tubo orotraqueal esté inflado $20-24$.

Como actores importantes del equipo de salud, se propone el presente artículo de reflexión que tiene como objetivo plantear el proceso de atención de enfermería durante las fases de planificación, preparación, pretratamiento y sedación con parálisis neuromuscular de la inducción de secuencia rápida que requieren los pacientes COVID-19 durante la intubación orotraqueal. Se sugiere que, para la atención de estos pacientes, el personal reciba entrenamientos previos preferiblemente en salas de simulación clínica o in situ ${ }^{21}$, que permita la adquisición de habilidades operativas en el manejo de los medicamentos requeridos para la intubación de pacientes con patología respiratoria por cualquier tipo de coronavirus ${ }^{25}$.

\section{Materiales y Métodos}

Artículo de reflexión sobre la inducción de secuencia rápida modificada utilizada en la intubación a pacientes COVID-19 con distrés respiratorio, para lo cual, se consultaron las bases de datos Scielo, Pudmed, Redalyc y Medline, ubicando consensos y protocolos nacionales e internacionales de manejo de la vía aérea, abordajes clínicos de pacientes COVID-19 y publicaciones de cuidado de enfermería para pacientes con enfermedad por COVID-19 en las fases críticas y no críticas publicados en los últimos cinco años en el idioma inglés y español. Se encontró un artículo de cuidado de enfermería al paciente con distrés respiratorio y dos artículos de enfermería que evidenciaban la clasificación de gravedad del síndrome de dificultad respiratoria del adulto con enfermedad por COVID-19. Los demás artículos consultados a través de las siguientes palabras clave: enfermería y cuidado, intubación orotraqueal, paciente COVID-19, infección por SARS-CoV-2, urgencia y emergencia, inducción de secuencia rápida a las cuales se le adicionaron boléanos y operadores de truncamiento, permitieron encontrar 857 artículos.

Se seleccionaron por título y resumen 124 específicos de intubación e inducción de secuencia rápida y al dar lectura completa de estos artículos, se logró obtener una muestra de 29 artículos, que permitieron describir el consenso de manejo de los medicamentos en la SIR a pacientes COVID-19. Se tuvo como marco metodológico los pasos del proceso de atención de enfermería en las fases de planificación y preparación, pretratamiento, sedación con parálisis neuromuscular y manejo postintubación, por ser las fases de la SIR en las que el personal de enfermería interviene de manera importante con la administración de la terapia farmacológica. El artículo cuenta la experiencia de los autores en el manejo de los medicamentos en estos pacientes durante la SIR y cómo desde el ejercicio profesional se ha llegado a las mejores prácticas de enfermería aplicando el proceso de atención de enfermería como una metodología para dirigir el cuidado.

\section{Resultados y Discusión}

En el proceso de inducción de secuencia rápida modificada en pacientes COVID-19, se hace necesario un efecto de sedación, relajación y analgesia que facilite la ventilación, se minimice 
el reflejo de tos y riesgo de aspiraciones y no se afecte el estado hemodinámico ${ }^{26}$. Para ello, conocer el mecanismo de acción de los medicamentos y los tiempos del efecto esperado que potencien la acción sedo analgésica y de relajación son de vital importancia.

Los medicamentos que se deben alistar en la fase de planificación y preparación son lidocaína y opiáceos de acción corta como el fentanilo los cuales disminuyen los efectos secundarios a la intubación evidenciados en hipotensión, bradicardia o taquicardia, aumento de la presión intracraneana y resistencia de la vía aérea ${ }^{8,11,20}$. Para la fase de inducción con parálisis neuromuscular que busca llevar a la inconsciencia y relajación muscular se utilizan sedantes (etomidato, ketamina, propofol, midazolam) y relajantes musculares (succinilcolina, rocuronio) ${ }^{23-26}$. Los vasopresores (norepinefrina, epinefrina, vasopresina y dopamina), también se tienen en cuenta en todas las fases para dar manejo a la inestabilidad hemodinámica que se podría presentar en la SIR modificada ${ }^{9,27}$. A continuación se hace referencia a las etapas del proceso de atención de enfermería como marco metodológico de presentación de los resultados encontrados en la revisión de la literatura.

\section{Valoración de enfermería}

En la valoración de enfermería se tuvieron en cuenta 19 artículos que permitieron describir los mecanismos de acción e incidencia de cada uno de estos en la función cardiovascular, cerebral y respiratoria. La lidocaína bloquea las fibras aferentes vágales, especialmente en el adulto mayor con COVID-19 para el manejo del laringoespasmo porque evita el reflejo de la tos durante la intubación orotraqueal. A su vez, la lidocaí-

En la valoración de enfermería se tuvieron en cuenta 19 artículos que permitieron describir los mecanismos de acción e incidencia de cada uno de estos en la función cardiovascular, cerebral y respiratoria especialmente. na disminuye el dolor durante la perfusión venosa de inductores de alta osmolaridad como el propofol ${ }^{19-21}$. Es importante valorar en estos pacientes la presencia de arritmias derivadas de tratamientos específicos para la infección por SARS-CoV-2 como cloroquina/hidroxicloroquina, azitromicina y lopinavir/ritonavir que ocasionan prolongación del intervalo QT, la lidocaína prolonga la repolarización por lo cual, no estaría indicada en la SIR ${ }^{28,29}$.

El fentanilo, opioide que produce el efecto analgésico durante la SIR, mantiene la estabilidad hemodinámica y no altera la función cardiovascular, sin embargo, es necesario valorar en los pacientes antes de su administración alguna condición de bradicardia ${ }^{16}$. A nivel cerebral, disminuye el consumo de oxígeno, el flujo sanguíneo y la presión intracraneana ${ }^{14,15}$. Tiene un efecto en el control de la respuesta simpática derivada de la maniobra de laringoscopia ${ }^{14}$, sin embargo, algunas revisiones de la literatura afirman que los opioides tienen el potencial de causar tos por lo cual, conviene administrarlos luego de la relajación muscular satisfactoria ${ }^{26}$.

En la fase de sedación con parálisis neuromuscular se procede a la utilización de medicamentos de inducción y de relajación neuromuscular. La inducción en el paciente COVID-19 tiene como objetivo llevar al paciente a la sedación y pérdida de la consciencia que facilite la intubación traqueal $^{14-16}$.

El etomidato tiene su efecto en el sistema nervioso central, de elección en pacientes COVID-19 que presentan inestabilidad hemodinámica, sin embargo, es un inductor que reduce el flujo sanguíneo cerebral por disminución del metabolismo y vasoconstricción cerebral. Este inductor 
obliga a revisar signos de disminución de la perfusión tisular cerebral, valores del dímero $\mathrm{D}$ y las plaquetas, puesto que, si el dímero $D$ está elevado y hay una reducción de plaquetas, estos pacientes son susceptibles a eventos cerebrovasculares agudos ${ }^{30}$.

La ketamina tiene su efecto sobre receptores de opioides en el sistema nervioso central llevando a analgesia, también útil en pacientes COVID-19 por su efecto de relajación en el musculo liso bronquial lo que facilita la intubación orotraqueal ${ }^{20,21,31}$, a su vez, en pacientes inestables hemodinámicamente es un inductor de elección porque recaptura noradrenalina, sin embargo, es importante valorar en los pacientes signos de isquemia miocárdica debido a que aumenta el consumo de oxígeno miocárdico ${ }^{24}$. La ketamina puede estimular enfermedades cardiovasculares por sus efectos simpaticomiméticos ${ }^{24}$. Es importante revisar en estos pacientes de infección por SARS-CoV-2, cambios electrocardiográficos sugestivos de isquemia miocárdica junto con biomarcadores cardiacos; el incremento en los niveles de troponina es común en pacientes con infecciones respiratorias agudas y la hipoxemia severa secundaria a disfunción respiratoria puede llevar a lesión miocárdica ${ }^{28}$. Se recomienda el uso de ketamina con rocuronio puesto que se minimiza el tiempo de apnea y riesgo de tos del paciente ${ }^{22}$.

El propofol es un inductor que ofrece protección cerebral, disminuye el consumo miocárdico y cerebral de oxígeno y reduce la presión arterial, por ello, en pacientes hemodinámicamente inestables no es ideal su uso. Es importante en el paciente COVID-19 valorar antes de su administración, la presencia de bradicardia e hipotensión arterial, así como cardiopatías dilatadas ya que estaría contraindicado su uso ${ }^{15}$. Se recomienda utilizar la combinación de propofol con lidocaína y fentanilo $20,21-24$.

El midazolam una benzodiacepina de acción sedante con efecto hipnótico y amnésico, es útil en pacientes con infección por SARS-CoV-2 en estados de ansiedad ${ }^{26}$, sin embargo, de especial cuidado durante la intubación por los efectos adversos de hipo y tos en pacientes que no estén bien relajados e hipotensión arterial en pacientes hipovolémicos ${ }^{14}$. Se recomienda su uso en pacientes COVID-19 hemodinámicamente inestables combinado con ketamina y fentanilo ${ }^{20}$. "El midazolam puede inducir apnea sin pérdida de la conciencia, lo cual dificulta el proceso de oxigenación durante la secuencia de inducción rápida"14. Una combinación de etomidato o ketamina y midazolam son recomendados en esta fase de inducción ${ }^{24}$.

La parálisis neuromuscular administrada al paciente COVID-19 inmediatamente después de la perdida de la conciencia ${ }^{26}$, busca una relajación muscular plena que facilite la intubación y disminuya la posibilidad de tos o movimientos durante la intubación orotraqueal ${ }^{21,31}$. El rocuronio es la primera elección porque reduce el riesgo de tos en comparación con la succinilcolina si los intentos de intubación son prolongados ${ }^{24}$. El uso del rocuronio obliga a prever el alistamiento de medicamentos que reviertan el bloqueo neuromuscular como el sugamandex ${ }^{21}$, una ciclodextrina que encapsula los relajantes musculares no despolarizantes en caso de que el paciente esté con una relajación neuromuscular de difícil intubación ${ }^{14,15}$. La succinilcolina es un bloqueador neuromuscular no despolarizante que compite con la acetilcolina en los receptores nicotínicos y su uso hace necesario revisar antecedentes de enfermedades del hígado ya que el metabolismo es hepático y niveles de potasio porque eleva sus niveles hasta en un $0.5 \mathrm{meq} / \mathrm{I}^{15,16,21,31}$ y la frecuencia cardiaca por la estimulación de receptores nicotínicos, que puede llevar a taquicardiaa $^{15,16}$. El atracurio se podría considerar en caso que el paciente no tolere la succinilcolina ${ }^{10}$. 
Los vasopresores tipo noradrenalina, epinefrina, vasopresina y dopamina son medicamentos necesarios para el manejo de la inestabilidad hemodinámica de los pacientes COVID-199. Los mecanismos que pueden ocasionar hipotensión en el paciente COVID-19 son cuatro, el primero es la vasoconstricción pulmonar que origina hipertensión pulmonar y disfunción del ventrículo derecho, el segundo es la tormenta de citoquinas que lleva vasodilatación y/o depresión miocárdica, la tercera son los eventos trombóticos especialmente el tromboembolismo pulmonar y como cuarta causa de hipotensión la presencia de choque cardiogénico originado por la miocarditis viral ${ }^{32}$. Lo anterior hace necesario que, durante la SIR se tenga disponible vasopresores que logren mantener una presión arterial media PAM $\geq 65 \mathrm{mmHg}$ para garantizar la perfusión tisular $^{33}$. Sin embargo, un estudio reciente sugiere que en adultos de 65 años o más, esta PAM en hipotensión vasodilatadora podría manejarse entre $60-65 \mathrm{mmHg}$ ya que equivale para estos pacientes a tener una PAM $\geq 65 \mathrm{mmHg}^{34}$. La norepinefrina se recomienda como el medicamento de primera línea en adultos para mantener la PAM en los rangos de perfusión tisular, se puede combinar con epinefrina o vasopresina para lograr el objetivo de PAM ${ }^{9}$. La norepinefrina en dosis bajas ayuda en la distribución del flujo pulmonar y en la diminución de la hipertensión pulmonar, si se requiere de dosis altas para el manejo hemodinámico, podría agravar la hipertensión pulmonar, por ello una combinación con vasopresina mejora la presión de perfusión tisular sin afectar la presión arterial pulmonar ${ }^{32}$. La dopamina se debe reservar para pacientes con bradiarritmia ${ }^{9}$ y dobutamina en disfunción miocárdica ${ }^{4}$.

\section{Diagnósticos de Enfermería}

Para establecer los diagnósticos de enfermería se tuvo como referencia bibliográfica específica de la disciplina de enfermería, el lenguaje estandarizado en los vínculos NANDA NOC NIC. En la fase de diagnóstico los profesionales de enfermería utilizan las habilidades de pensamiento crítico que conduzcan a la planeación e intervenciones de cuidado al paciente COVID-19 que requiere intubación con SIR y al personal de salud que realiza este procedimiento. En la tabla 1 se proponen diagnósticos de enfermería NANDA y resultados de enfermería esperados $\mathrm{NOC}^{35}$.

Para establecer los diagnósticos de enfermería se tuvo como referencia bibliográfica específica de la disciplina de enfermería, el lenguaje estandarizado en los vínculos NANDA NOC NIC.

Tabla 1. Diagnósticos de Enfermería NANDA y Resultados Esperados NOC

\begin{tabular}{clcl}
\hline $\begin{array}{c}\text { Código } \\
\text { NANDA }\end{array}$ & \multicolumn{1}{c}{ Diagnóstico de Enfermería } & $\begin{array}{c}\text { Código } \\
\text { NOC }\end{array}$ & \multicolumn{1}{c}{ Resultados Esperados } \\
\hline 00147 & Ansiedad ante la muerte & 1201 & Esperanza \\
00033 & Deterioro de la ventilación espontánea & 402 & Estado respiratorio: intercambio gaseoso \\
& & 403 & Estado respiratorio: ventilación \\
00180 & Riesgo de contaminación & 1911 & Conductas de seguridad personal \\
\hline
\end{tabular}

Fuente: elaboración propia

\section{Planeación de enfermería}

En esta fase, se plasma referencias bibliográficas que evidencian el cuidado de enfermería en la administración de medicamentos, así como la experiencia de los autores como integrantes del personal de salud que participa en la intubación e inducción de secuencia rápida a pacientes COVID-19, lo que hace necesario revisar, el alistamiento de los medicamentos que serán usados 
en la SIR, las condiciones del acceso venoso y el espacio físico de trabajo para la preparación y la administración de los medicamentos.

El alistamiento de los fármacos obliga conocer la presentación, dosis, concentración de preparación de medicamentos sugeridos en la SIR al paciente con infección por coronavirus COVID-19. Tabla 2. Los medicamentos que se administran en perfusión bolo, se recomienda envasarlos en jeringas de $5 \mathrm{ml}$ o $10 \mathrm{ml}$, sin diluir en ningún tipo de solución y marcarlos con el nombre y la concentración por mililitro (ml). En la figura 1 se ejemplifica la preparación de estos medicamentos. Es importante alistar una jeringa de $20 \mathrm{ml}$ con una solución isotónica que permita el arrastre del medicamento a través del tubo de perfusión que se encuentra conectado al acceso venoso para lograr el nivel plasmático en los tiempos esperados y con ello alcanzar el efecto ${ }^{36}$.
En esta fase, se plasma referencias bibliográficas que evidencian el cuidado de enfermería en la administración de medicamentos, así como la experiencia de los autores como integrantes del personal de salud que participa en la intubación e inducción de secuencia rápida a pacientes Covid-19, lo que hace necesario revisar, el alistamiento de los medicamentos que serán usados en la SIR, las condiciones del acceso venoso y el espacio físico de trabajo para la preparación y la administración de los medicamentos.
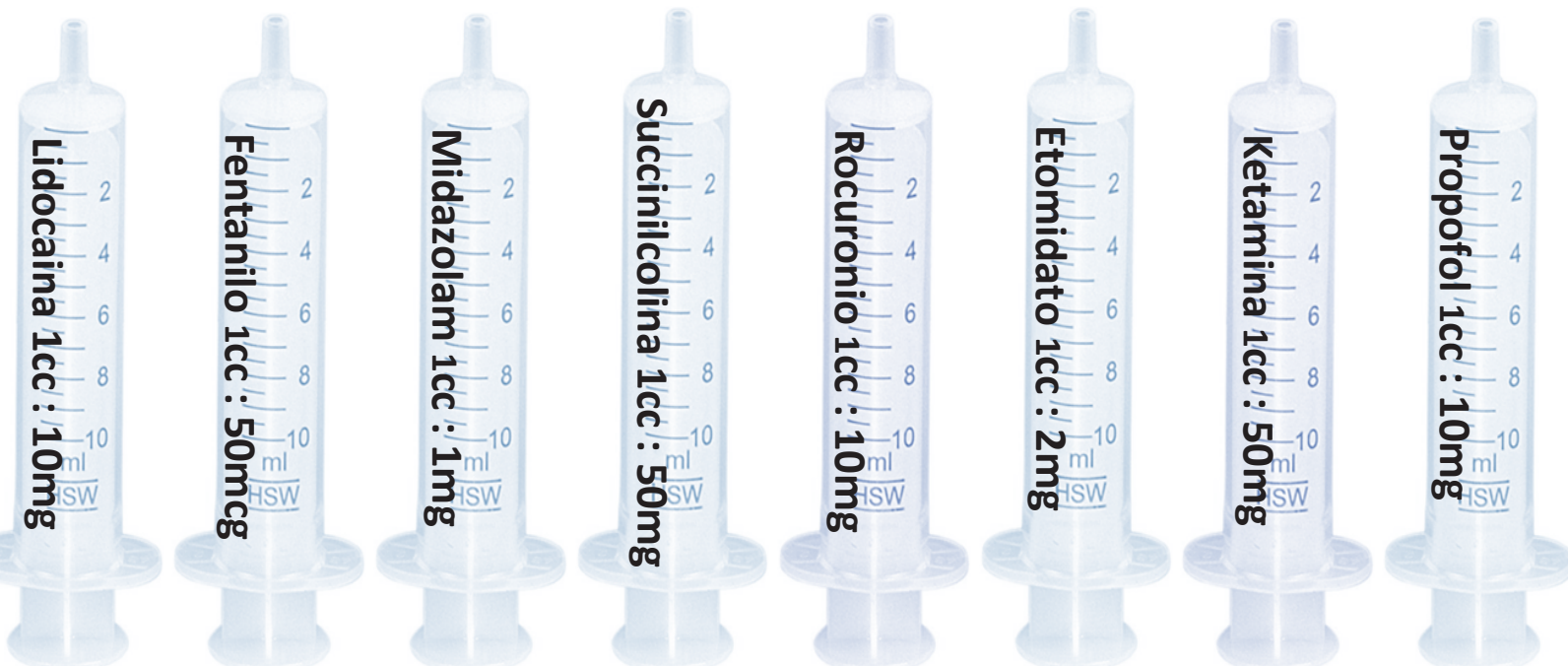

Figura 1 Alistamiento de medicamentos de perfusión bolo durante la SIR en pacientes COVID-19 Fuente: elaboración propia

En esta fase de planeación es importante hacer la verificación de la permeabilidad del acceso venoso del paciente el cual puede ser periférico o central. Sin embargo, en caso de no ser posible el acceso venoso, el uso de una aguja intraósea podría ser necesaria. Loubani y Green citados por la OMS, afirman que los vasopresores se administran de manera más segura a través de un catéter venoso central a un ritmo estrictamente controlado, pero también es posible administrarlos de manera segura a través de una vena periférica y una aguja intraósea ${ }^{37}$. El acceso venoso permeable forma parte de acrónimos de las fases de preparación para la intubación de los pacientes COVID 19; una experiencia en Wuhan recomienda el acrónimo OH-MS. MAID (oxigeno, ayuda, monitor, succión, maquinas, vía aérea, acceso intravenoso y drogas) ${ }^{26}$.

El área de preparación de los medicamentos debe ser iluminado, ventilado y seguro lo cual coincide con los principios seguro, apropiado y oportuno descritos en el Consenso de Guías para el Manejo de la Vía Aérea en pacientes con COVID-19²2. Este consenso sugiere tener disponible un 
carro de intubación traqueal para pacientes que padecen COVID 19, que sea fácil de transportar y descontaminar después de su uso ${ }^{22}$, el cual debe tener un dispensador de alcohol glicerinado para la higienización de los guantes y recipientes de residuos hospitalarios para manejo de material cortopunzante y no cortopunzante. Debe existir un plan de ubicación del personal para la intubación orotraqueal de un paciente COVID-1922,38, los cuales se han adaptado de guías de manejo de intubación de pacientes adultos $\operatorname{críticos}^{31}$ y que proponen un mínimo equipo de trabajo reducido a 3 personas dentro de la sala: quien se encargue de la intubación, la persona que asiste la vía aérea y/o cricotiroidectomia y el encargado de los medicamentos-monitor 22 , tal y como se representa en la Figura 2.

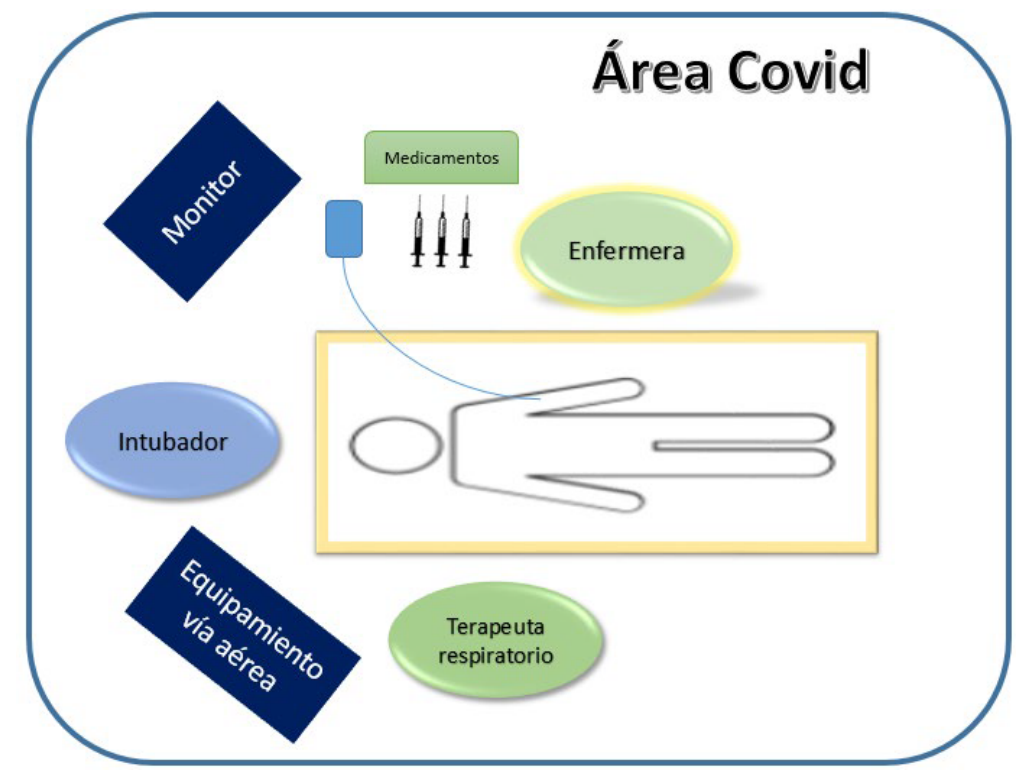

Figura 2. Ubicación del personal de salud en área COVID-19. Fuente: elaboración propia adaptado de fuente consultada ${ }^{22,31}$

\section{Intervención de enfermería}

En esta fase de intervención se pretende hacer un diálogo entre la literatura consultada y la experiencia de los autores haciendo una propuesta de organización y tiempos en la administración de los medicamentos que den respuesta a la inducción de secuencia rápida en la intubación orotraqueal a pacientes Covid-19. La secuencia inicia con la administración del pretratamiento utilizando fentanilo y lidocaína 3 minutos antes de los inductores, es decir, durante la fase de preoxigenación que tiene un tiempo estimado de 5 minutos ${ }^{22}$, considerando el vasopresor de ser necesario en la inestabilidad hemodinámica, seguido de la inducción con ketamina en caso de choque o broncoespasmo, midazolam en caso de cardiopatía o etomidato en caso de choque; inmediatamente después que el paciente tenga pérdida de la conciencia, viene la parálisis neuromuscular con rocuronio o succinilcolina ${ }^{20,24,26}$; se cuenta en segundos el tiempo de inicio del efecto para el rocuronio 60 a 75 segundos y para la succinilcolina 45 segundos ${ }^{16}$ y viene la intubación orotraqueal. Tabla 2. La experiencia en Wuhan refiere que luego de una preoxigenación satisfactoria, iniciar con midazolam para disminuir la ansiedad y continuar con la lidocaína para suprimir la tos, etomidato en pacientes inestables o propofol en pacientes

En esta fase de intervención se pretende hacer un diálogo entre la literatura consultada y la experiencia de los autores haciendo una propuesta de organización y tiempos en la administración de los medicamentos que den respuesta a la inducción de secuencia rápida en la intubación orotraqueal a pacientes Covid-19. 
estables hemodinámicamente y finalizar con rocuronio o succinilcolina inmediatamente después de la perdida de la conciencia. Se recomienda el uso del fentanilo para suprimir el reflejo laríngeo durante la intubación luego de la relajación muscular satisfactoria debido a que los opioides pueden causar $\operatorname{tos}^{26}$. Los tiempos durante la SIR pueden variar por dos razones, la primera es la situación clínica de los pacientes cuyos niveles de oxigenación tienden a bajar a pesar de la preoxigenación, lo que obliga a actuar rápidamente para lograr mejorar la saturación de oxígeno y la segunda es el nivel de ansiedad de personal de salud al riesgo de contaminación, lo que imposibilita seguir de manera estricta estos tiempos; recomendamos tener un reloj con segundero en la pared visible a enfermería que permita lograr una aproximación de los tiempos durante la SIR.

La SIR inversa en la que se inicia con la administración de los bloqueantes neuromusculares antes que los inductores para lograr tiempos de intubación más cortos, es un tipo de secuencia utilizada en aquellos pacientes con menor reserva fisiológica como lo es el síndrome de dificultad respiratoria ${ }^{39}$. Se coloca el rocuronio cuya acción inicia a partir de los 60 a 75 segundos y a los 15 segundos de la administración del rocuronio, se infunde la ketamina cuya acción inicia a los 45 segundos. Lo que se espera es un efecto de relajación y sedación entre los 45 y 75 segundos siendo el momento oportuno para la intubación orotraqueal como lo muestra la figura 3.

La SIR inversa podría reducir en 15 segundos el tiempo de llegar a la intubación lo cual busca cumplir con el objetivo de asegurar la vía aérea del paciente con sospecha de COVID-19 en los 60 segundos posteriores a la administración del relajante muscular acortando el tiempo de exposición del personal de salud a este procedimiento. Esta secuencia inversa podría ser utilizada por personal médico entrenado en este tipo de secuencia, sin embargo, en nuestra experiencia asistencial, es poco frecuente su uso en los servicios de urgencias y hace necesario que el personal de enfermería tenga claras las variaciones en la administración de los medicamentos, con el fin de garantizar la seguridad del paciente y del personal de salud, en términos de minimizar los tiempos de exposición y riesgo de contaminación.

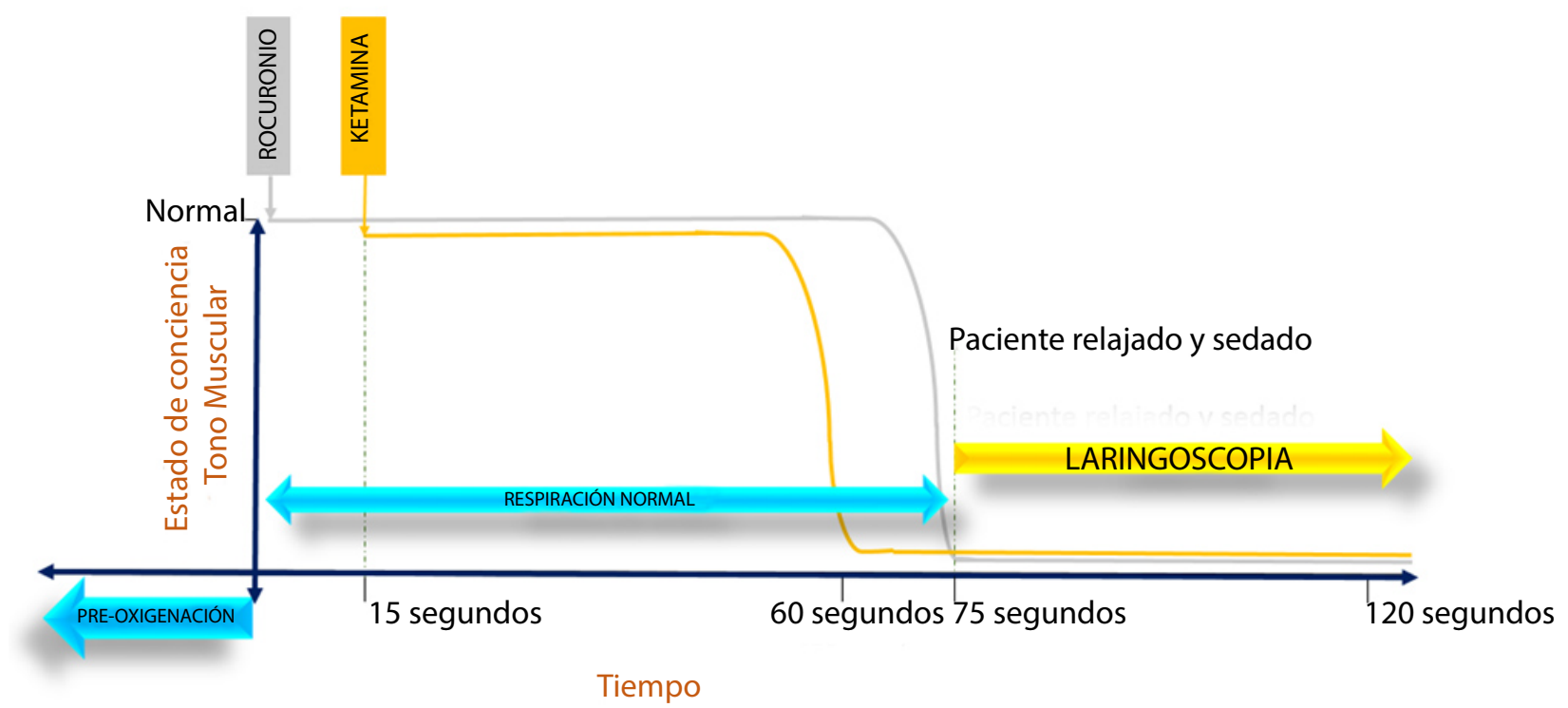

Figura 3 Secuencia de inducción rápida inversa.

Fuente: elaboración propia adaptado de fuente consultada ${ }^{40}$ 
En esta fase de intervención de enfermería, se debe asegurar que los medicamentos se administren a través de los puertos en $\mathrm{Y}$ que tienen los equipos de venoclisis conectados a los accesos venosos permeables, asegurando que la administración sea en perfusión bolo en un tiempo de 30 a 60 segundos $^{36}$. El fentanilo por su efecto adverso de tórax en leña cuando se administra en bolos rápidos ${ }^{41}$, se recomienda colocarlo en un tiempo mínimo de 60 segundos. Una vez se administren los medicamentos, es necesario hacer el lavado del equipo de venoclisis con 20 mililitros de solución isotónica.

\section{Evaluación de enfermería}

Se revisan 6 artículos que, de manera relevante, proponen la sedo analgesia y relajación que requieren los pacientes Covid-19 una vez se le ha

Se revisan 6 artículos que, de manera relevante, proponen la sedo analgesia y relajación que requieren los pacientes COVID-19 una vez se le ha realizado la intubación. A su vez, establecen la evaluación de la función hemodinámica y su realizado la intubación. A su vez, establecen la evaluación de la función hemodinámica y su respectivo manejo en la inestabilidad. respectivo manejo en la inestabilidad. Los medicamentos en perfusión continua recomendados para la sedación son el propofol o la dexmedetomidina antes que las benzodiacepinas ${ }^{42}$. El haloperidol o antipsicóticos para el tratamiento del delirio, se deben utilizar en periodos cortos de tiempo y no de manera rutinaria ${ }^{42}$. Como sedo-analgesia, los medicamentos opioides morfina y fentanilo son los indicados ${ }^{43}$, sin embargo, la perfusión continua ha demostrado que aumentan los días de ventilación y la estancia en las unidades de cuidado intensivo, por ello, el uso de remifentanilo es una opción porque no se acumula, lo que permite una recuperación rápida ${ }^{43}$.

Los relajantes neuromusculares se requieren para evitar la asincronía del paciente con la ventilación mecánica y mejorar la oxigenación, para lo cual, se sugiere que sea en perfusión bolo y reservar la perfusión continua, que no debe ser por más de 48 horas, a pacientes que no han logrado la sincronía con la ventilación mecánica, que requieran una sedación muy profunda, que deban colocarse en posición prono, que tengan un indice de oxigenación de relación Presión arterial de oxígeno y la fracción inspirada de oxígeno Pao2/FIO2 $<150$ o requieran PEEP $>14 \mathrm{~cm}$ $\mathrm{H} 2 \mathrm{O}$; los más recomendados son succinilcolina, atracurio o vecuronio ${ }^{42,44}$.

En caso de inestabilidad hemodinámica, el uso de soluciones isotónicas es recomendada en volúmenes bajos especialmente en disfunción del ventrículo derecho ${ }^{42}$. En un metaanálisis, sugieren el uso de vasoactivos a dosis bajas para alcanzar presión arterial media entre 60 y $65 \mathrm{mmHg}$, siendo la noradrenalina el medicamento de elección, sin embargo, si la hipoperfusión tisular persiste a pesar de la reanimación hídrica en conjunto con la administración de noradrenalina, se debe considerar el manejo concomitante con dopamina antes que aumentar la dosis de noradrenalina ${ }^{33}$ y en disfunción miocárdica utilizar dobutamina ${ }^{4,9}$. En la tabla 2 se propone la perfusión continua de la sedo-analgesia pos-intubación y los vasopresores que podrían ser utilizados durante la intubación y pos-intubación; para estos vasopresores, se sugiere diluirlos en solución salina normal al 0.9\% puesto que en pacientes COVID-19 se debe evitar el uso de soluciones hipotónicas ${ }^{42}$. 
Tabla No. 2 Medicamentos en la SIR en perfusión bolo y perfusión continua, tiempos de acción y tiempos de administración.

\begin{tabular}{|c|c|c|c|c|c|c|c|}
\hline Medicamento & Presentación & $\begin{array}{l}\text { Perfusión } \\
\text { bolo* } \\
\text { dosis }^{20,21,24}\end{array}$ & Concentración & $\begin{array}{l}\text { Tiempo de } \\
\text { inicio del } \\
\text { efecto }^{16,46}\end{array}$ & $\begin{array}{l}\text { Duración } \\
\text { del } \\
\text { efecto }^{16,46}\end{array}$ & $\begin{array}{l}\text { Preparación } \\
\text { de la mezcla } \\
\text { perfusión } \\
\text { continua** }\end{array}$ & Cuidado de enfermería \\
\hline $\begin{array}{l}\text { Lidocaína } 1 \% \\
\text { sin epinefrina }\end{array}$ & $10 \mathrm{mg} / \mathrm{ml}$ & $1-1,5 \mathrm{mg} / \mathrm{kg}$ & {$[10 \mathrm{mg} / \mathrm{ml}]$} & Inmediato & 10 a $20 \mathrm{~min}$ & $\begin{array}{l}\text { No se } \\
\text { recomienda }\end{array}$ & $\begin{array}{l}\text { Valorar presencia de arritmias } \\
\text { derivadas de intervalo QT prolonga- } \\
\text { do o torsión de puntas }{ }^{28,29} \text {. }\end{array}$ \\
\hline Fentanilo & $0.5 \mathrm{mg} / 10 \mathrm{ml}$ & $1-2 \mathrm{mcg} / \mathrm{kg}$ & {$[50 \mathrm{mcg} / \mathrm{ml}]$} & 2 a 3 minutos & 30 a $60 \mathrm{~min}$ & $\begin{array}{l}\text { SSN0.9\% 80ml } \\
\text { más } 20 \mathrm{ml} \mathrm{de} \\
\text { fentanilo } \\
\text { [10mcg/ml] }\end{array}$ & $\begin{array}{l}\text { Valorar presencia de bradiarritmia }{ }^{16} \text {. } \\
\text { Administración lenta para prevenir } \\
\text { rigidez muscular que dificulte la } \\
\text { expansión torácica }{ }^{39} \text {. }\end{array}$ \\
\hline
\end{tabular}
No se Revisar signos de disminución de la recomienda perfusión cerebral conjuntamente con el dímero $D$ elevado y trombocitopenia $^{30}$

\begin{tabular}{|c|c|c|c|c|c|c|c|}
\hline Ketamina & $500 \mathrm{mg} / 10 \mathrm{ml}$ & $1-2 \mathrm{mg} / \mathrm{kg}$ & {$[50 \mathrm{mg} / \mathrm{ml}]$} & $\begin{array}{l}45 \text { a } 60 \\
\text { segundos }\end{array}$ & 15 a $20 \mathrm{~min}$. & $\begin{array}{l}\text { No se } \\
\text { recomienda }\end{array}$ & $\begin{array}{l}\text { Valorar signos de hipertensión } \\
\text { endocraneana. } \\
\text { Valorar signos de isquemia miocár- } \\
\text { dica }^{24} \text {. }\end{array}$ \\
\hline Propofol & $200 \mathrm{mg} / 20 \mathrm{ml}$ & $1-2 \mathrm{mg} / \mathrm{kg}$ & {$[10 \mathrm{mg} / \mathrm{ml}]$} & $\begin{array}{c}15 \text { a } 45 \\
\text { segundos }\end{array}$ & 5 a $10 \mathrm{~min}$. & $\begin{array}{l}30 \text { a } 50 \mathrm{ml} \text { de } \\
\text { propofol sin } \\
\text { diluir } \\
{[10 \mathrm{mg} / \mathrm{ml}]}\end{array}$ & $\begin{array}{l}\text { Valorar antes de su administración, } \\
\text { la presencia de bradicardia e } \\
\text { hipotensión arterial, así como } \\
\text { cardiopatías dilatadas }^{15,46} \text {. }\end{array}$ \\
\hline Midazolam & $5 \mathrm{mg} / 5 \mathrm{ml} 0$ & $0.05-0.1 \mathrm{mg} / \mathrm{kg}$ & {$[1 \mathrm{mg} / \mathrm{ml}]$} & $\begin{array}{l}60 \text { a } 90 \\
\text { segundos } \\
\text { Duración del } \\
\text { efecto: de }\end{array}$ & 15 a $30 \mathrm{~min}$. & $\begin{array}{l}50 \mathrm{ml} \mathrm{o} \\
100 \mathrm{ml} \mathrm{de} \\
\text { midazolam } \\
\text { sin diluir } \\
{[1 \mathrm{mg} / \mathrm{ml}]}\end{array}$ & $\begin{array}{l}\text { Valorar hipotensión en pacientes } \\
\text { hipovolémicos y revisar la presencia } \\
\text { de apnea sin pérdida de la concien- } \\
\text { cia }^{14} \text {. }\end{array}$ \\
\hline Rocuronio & $50 \mathrm{mg} / 5 \mathrm{ml}$ & $1-2 \mathrm{mg} / \mathrm{kg}$ & {$[10 \mathrm{mg} / 1 \mathrm{ml}]$} & $\begin{array}{c}60 \text { a } 75 \\
\text { segundos }\end{array}$ & 40 a $60 \mathrm{~min}$ & $\begin{array}{l}\text { SSN0.9\% } \\
45 \mathrm{ml} \text { más } \\
5 \mathrm{ml} \text { de } \\
\text { rocuronio } \\
{[1 \mathrm{mg} / \mathrm{ml}]}\end{array}$ & $\begin{array}{l}\text { Alistar medicamentos que reviertan } \\
\text { el bloqueo neuromuscular como el } \\
\text { sugamandex }{ }^{15} \text {, a su vez, revisar la } \\
\text { presencia de alergia luego de su } \\
\text { administración }{ }^{46} \text {. }\end{array}$ \\
\hline Succinilcolina & $100 \mathrm{mg} / 2 \mathrm{ml}$ & $1-1.5 \mathrm{mg} / \mathrm{kg}$ & {$[50 \mathrm{mg} / \mathrm{ml}]$} & 45 segundos & 6 a $10 \mathrm{~min}$ & $\begin{array}{c}\text { SSN0.9\% } \\
90 \mathrm{ml} \text { más } \\
10 \mathrm{ml} \mathrm{de} \\
\text { succinilcolina } \\
{[5 \mathrm{mg} / \mathrm{ml}]}\end{array}$ & $\begin{array}{l}\text { Valorar antecedentes de enferme- } \\
\text { dad hepática, hipertermia maligna, } \\
\text { niveles de potasio porque produce } \\
\text { hipercalcemia y revisar presencia de } \\
\text { taquicardia porque la podría } \\
\text { empeorar }{ }^{15,16,21,31,46} \text {. }\end{array}$ \\
\hline
\end{tabular}

\begin{tabular}{|c|c|c|c|c|}
\hline \multicolumn{5}{|c|}{ Vasopresores } \\
\hline Medicamento & Presentación & $\begin{array}{l}\text { Perfusión continua** } \\
\text { dosis }^{48}\end{array}$ & $\begin{array}{c}\text { Preparación de la mezcla } \\
\text { perfusión continua** }\end{array}$ & Cuidado de enfermería $^{47,48}$ \\
\hline Noradrenalina & $4 \mathrm{mg} / 4 \mathrm{ml}$ & $0.05-1.5 \mathrm{mcg} / \mathrm{kg} / \mathrm{min}$ & $\begin{array}{l}\text { SSN0.9\% 96ml más } 4 \mathrm{ml} \text { de } \\
\text { noradrenalina }[40 \mathrm{mcg} / \mathrm{ml}]\end{array}$ & $\begin{array}{l}\text { Monitorizar la presión de la arteria pulmonar } \\
\text { porque las dosis altas pueden llevar a hiperten- } \\
\text { sión pulmonar e incremento de la presión } \\
\text { venosa central. }\end{array}$ \\
\hline Dopamina & $200 \mathrm{mg} / 5 \mathrm{ml}$ & $\begin{array}{l}\text { Dosis beta inotrópica } \\
>5-10 \mathrm{mcgrs} / \mathrm{kg} / \mathrm{min}\end{array}$ & $\begin{array}{l}\text { SSN0.9\% } 245 \mathrm{ml} \text { más } 5 \mathrm{ml} \text { de } \\
\text { dopamina }[800 \mathrm{mcg} / \mathrm{ml}]\end{array}$ & $\begin{array}{l}\text { Monitorizar presencia de hipertensión pulmo- } \\
\text { nar, revisar aparición de taquiarritmias especial- } \\
\text { mente cuando se combina con dobutamina. }\end{array}$ \\
\hline Dobutamina & $250 \mathrm{mg} / 20 \mathrm{ml}$ & $\begin{array}{l}\text { Dosis inotrópica } \\
>5-20 \mathrm{mcgrs} / \mathrm{kg} / \mathrm{min}\end{array}$ & $\begin{array}{l}\text { SSN0.9\% } 230 \mathrm{ml} \text { más } 20 \mathrm{ml} \text { de } \\
\text { dobutamina }[1000 \mathrm{mcg} / \mathrm{ml}]\end{array}$ & $\begin{array}{l}\text { Revisar que la infusión no se prolongue por más } \\
\text { de } 72 \text { horas para que no se genere taquifilaxia } \\
\text { por descenso en la regulación de los receptores } \\
\text { beta. }\end{array}$ \\
\hline Vasopresina & $20 \mathrm{U} / 1 \mathrm{ml}$ & 1-2unidades/hora & $\begin{array}{l}\text { SSN0.9\% 99ml más } 1 \mathrm{ml} \text { de } \\
\text { vasopresina }[0.2 \mathrm{U} / \mathrm{ml}]\end{array}$ & $\begin{array}{l}\text { Valorar signos de disminución de la perfusión } \\
\text { distal o necrosis resultado de vasoconstricción } \\
\text { periférica especialmente si se combina con } \\
\text { noradrenalina. }\end{array}$ \\
\hline
\end{tabular}

*Perfusión bolo: medicamentos que en la SIR se administran entre los 30 y 60 segundos.

**Perfusión continua: medicamentos que en la SIR se administran hora a hora. 


\section{Conclusiones}

Aplicar el proceso de atención de enfermería en la inducción de secuencia rápida durante las fases de planificación, preparación, pretratamiento y sedación con parálisis neuromuscular, contribuye con la organización de los medicamentos requeridos para la intubación orotraqueal, lo cual, lleva a disminuir la ansiedad del paciente y el riesgo de contaminación del personal de salud. En la valoración es relevante tener en cuenta el mecanismo de acción de cada uno de los medicamentos y los aspectos fisiopatológicos del paciente que permitan determinar la indicación o contraindicación de los fármacos, en la fase de diagnóstico se busca identificar problemas reales o potenciales para el paciente y el personal de salud durante el pretratamiento y sedación con parálisis neuromuscular; la planeación hace necesario revisar el alistamiento de cada uno de los medicamentos, las condiciones de acceso venoso y el espacio físico de trabajo del personal de enfermería; la intervención aborda la secuencia y los tiempos de administración de los medicamentos y por último, la evaluación permite revisar la sedo-analgesia, relajación y estabilidad hemodinámica luego de la intubación orotraqueal.

Declaración de conflicto de intereses: Los autores no declaran ningún conflicto de interés.

Financiación: Recursos propios de los autores.

\section{Referencias}

1. Chica C, Peña L, Villamarín H, Moreno J, Rodríguez L, Lozano W, et al. Cuidado respiratorio en COVID-19. Acta Colombiana de Cuidado Intensivo. 2020;20(2):108-117. https://doi.org/10.1016/j.acci.2020.04.001

2. Gómez C, Peñuelas O, Lujan M, Santaolalla C, Masa J, García J, et al. Recomendaciones de consenso respecto al soporte respiratorio no invasivo en el paciente adulto con insuficiencia respiratoria aguda secundaria a infección por SARS-CoV-2. Archivos de Bronconeumología. 2020;56(2):11-18. https://doi.org/10.1016/j.arbres.2020.03.005

3. Enríquez M, Fernández M, Fontan G. Guía de actuación enfermera ante COVID-19. 2020. [Consultado el 15 de junio de 2020]; 3-4. Disponible en: https://www.consejogeneralenfermeria.org/images/coronavirus/documentos/GUIA\%20DE\%20ACTUACIO\%CC\%81 N\%20ENFERMERA\%20FRENTE\%20AL\%20COVID.pdf

4. Gobierno de España. Ministerio de Sanidad. Consenso de España. Manejo Clínico del COVID-19: atención hospitalaria. 2020. [Consultado el 15 de junio de 2020];6-8. Disponible en: https://fundacionio.com/wp-content/uploads/2020/03/Protocolo_manejo_clinico_tto_ COVID-19.pdf

5. Phua J, Weng L, Ling L, Egi M, Lim C, Divatia J, et al. Intensive care management of coronavirus disease 2019 (COVID-19): challenges and recommendations. Lancet Respir Med. 2020;8:506-17. https://doi.org/10.1016/S2213-2600(20)30161-2

6. Barrantes F, Vargas Z. Guía de cuidados de enfermería para el decúbito prono en Síndrome de Distrés Respiratorio Agudo asociado a COVID-19: Revisión integrativa. Revista Médica de Costa Rica. 2020;58-67. Disponible en file:///E:/docimuntos\%20c/Downloads/293-592-1-PB.pdf

7. Xu Z, Shi L, Wang Y, Zhang J, Huang L, Zhang C, et al. Pathological findings of COVID-19 associated with acute respiratory distress syndrome. Lancet Respir Med. 2020;8:420-22. https://doi.org/10.1016/S2213-2600(20)30076-X

8. Yi Y, Lagninton P, Ye S, Li E, Xu R. COVID-19: What Has Bee Learned and to Be Learned About the Novel Coronavirus Disease. Int J Biol Sci. 2020;16(10):1753-1766. doi: 10.7150/ijbs.45134 
9. World Health Organization. Clinical management of COVID-19. Interim guidance 27 May 2020. [Consultado el 15 de junio de 2020]; 22-28. Disponible en: https://apps.who.int/iris/ bitstream/handle/10665/332196/WHO-2019-nCoV-clinical-2020.5-eng.pdf?sequence=1\&isAllowed=y

10.Staff safety during emergency airway management for COVID-19 in Hong Kong. Published Online February 24, 2020. https://doi.org/10.1016/S2213-2600(20)30084-9

11.Navarro G, Padilla J. Manejo de la Vía Aérea en pacientes con COVID-19. Revista Médica de Costa Rica. 2020;58-67. file:///E:/docimuntos\%20c/Downloads/297-596-1-PB\%20(1).pdf

12.Peng P, Leung Ho P, Hota S. COVID-19 and the anaesthetist: a especial series. Outbreak of a new cornonavirus: what anaesthetists should know. British Journal of Anaesthesia. 2020;124(5):497-501. https://doi.org/10.1016/j.bja.2020.02.008

13.Navarro G, Sandoval C, Quintero K, Pérez L. Valoración y manejo de la vía aérea en paciente con enfermedad COVID-19 (Parte II). Revista electrónica de Anestesia. 2020; Disponible en: https://anestesiar.org/2020/valoracion-y-manejo-de-la-via-aerea-en-paciente-con-enfermedad-covid-19-parte-ii/

14.Pérez P, Moreno A, Gempeler F. Guía para la secuencia de inducción e intubación rápida en el servicio de emergencias. Universitas Medica. 2013;175-198.

https://doi.org/10.11144/Javeriana.umed54-2.gpsi

15.Zamarrón E, Pérez O, Díaz M, Sánchez J, Soriano R, Guerrero M, et al. Secuencia de inducción rápida en paciente crítico. Acta Colomb Cuid Intensivo. 2020;20(1):23-32.

https://doi.org/10.1016/j.acci.2019.09.002

16.Almarales J, Saavedra M, Salcedo O, Wady D, Morales J, Quijano C, et al. Inducción de secuencia rápida para intubación orotraqueal en Urgencias. Repert Med Cir. 2016; 25(4):210218. https://doi.org/10.1016/j.reper.2016.11.009

17.Gómez J, González A, Patarroyo M, Rodríguez A, Alvarez C, Suárez J. et al. Consenso Colombiano de Atención, Diagnóstico y Manejo de la Infección por SARCS-CoV-2/COVID-19 en Establecimientos de Atención de la Salud. Recomendaciones Basadas en Consenso de Expertos e Informadas en la Evidencia. Revista de la Asociación Colombiana de Infectología Infectio. 2020; portada. Disponible en:

http://www.revistainfectio.org/index.php/infectio/article/view/872

18.Vega A, Ayala M. Nuevo Coronavirus (COVID-19), historia, medidas de protección para la población y actuación del personal de salud. Grupo de Trabajo Sectorial de Enfermería de Unidades de Infecciosos. Red Internacional de Enfermería International Nursing Network. 2020. Disponible en: http://inursingn.com/wp-content/uploads/2020/03/infecciosos2.pdf

19.Garduño A, Guido R, Guizar M, Acosta V, Domínguez G, Alvarez G. Manejo perioperatorio de paciente con COVID-19. Revista Mexicana de Anestesiología. 2020;43(2):109-120. https://doi.org/10.35366/92869

20.Rebellón D, Caicedo E, Méndez Y. Recomendaciones para el abordaje de pacientes con COVID-19 en Boyacá. Grupo de Investigación en Epidemiología Clínica de Colombia Universidad Pedagógica y Tecnológica de Colombia. 2020. 143-153. Disponible en: file:///E:/docimuntos\%20c/Downloads/LibroCOVIDBoyaca.pdf

21.Mingzhang $Z$, Yuguang $H$, Wuhua $M$, Zhanggang $X$, Jiaqiang Z, Yahong $G$, et al. Expert Recommendations for Tracheal Intubation in Critically ill Patients with Noval Coronavirus Disease 2019. Chinese Medical Sciences Journal. 2020; 4-5. Disponible en:

file:///E:/docimuntos\%20c/Downloads/Expert\%20Recommendations\%20for\%20Tracheal\%20Intubation\%20in\%20Critically\%20ill\%20Patients\%20with\%20Noval\%20Coronavirus\%20Disease\%202019\%20(3).pdf

22.Cook T.M, El-Boghdadly K, Mc Guire B, McNarry A, Patel A, Higgs A. Consenso de Guías para el Manejo de la Vía Aérea en Pacientes con Covid-19. Guías de la Sociedad de Vía Aérea Difícil (DAS), la Asociación de Anestesistas, la Sociedad de Terapia Intensiva, la Facultad de 
Medicina de Cuidado Crítico y el Real Colegio de Anestesiólogos. Anaesthesia 2020. Disponible en: https://eva-la.org/wp-content/uploads/2020/04/Guias-de-manejo-VA-COVID-19Version-Larga.pdf

23.Accini J, Beltrán N, Nieto V, Ramos E. Declaración de Consenso en Medicina Critica para la Atención Multidisciplinaria del Paciente con Sospecha o Confirmación Diagnóstica de COVID-19. Asociación Colombiana de Medicina Critica y Cuidado Intensivo. 2020. Disponible en: https://www.amci.org.co/images/consenso/Capitulo_6_Intubacion_orotraqueal_segura. pdf

24.Yao W, Wang T, Jiang B, Gao F, Wang Li, Zheng $\mathbf{H}$, et al. Emergency tracheal intubation in 202 patients with COVID-19 in Wuhan, China: lessons learnt and international expert recomendatios. British Journal of Anaesthesia. 2020;125(1):e28-e37.

https://doi.org/10.1016/j.bja.2020.03.026

25.Sorbello M, Boghdadly K, DiGiacinto I, Cataldo R, Esposito C, Falcetta S, et al. The Italian coronavirus disease 2019 outbreak: recommendations from clinical practice. Anaesthesia 2020;(75):724-732. https://doi.org/10.1111/anae.15049

26.Meng L, Qiu H, Wan Li, Ai Y, Zhanggang X, Qulian G, et al. Intubation and Ventilation amid the COVID-19 Outbreak. Anesthesiology 2020;132(6):1317-32.

https://doi.org/10.1097/ALN.0000000000003296

27.Hernandez S, Saavedra J, Zamarron E, Perez O, Figueroa A, Guerrero M. et al. Protocolo de atención para COVID-19 (SARS.CoV-2) de la Sociedad Mexicana de Medicina de Emergencias. Sociedad Mexicana de Emergencia, A.C. 2020;39-41. Disponible en:

file:///E:/docimuntos\%20c/Downloads/GuiaCOVID19SMME.pdf

28.Figueroa J, Salas D, Cabrera J, Alvarado C, Buitrago A. COVID-19 y enfermedad cardiovascular. Revista Colombiana de Cardiología. In Press, Journal Pre-proof. 2020.

https://doi.org/10.1016/j.rccar.2020.04.004

29.García R, Rivero L, Aroche R, Aldama L, Hernández M. COVID-19: en torno al sistema cardiovascular. Anales Academia de Ciencias de Cuba. 2020. Disponible en:

http://www.revistaccuba.sld.cu/index.php/revacc/article/view/782/820

30.Aguayo S. et al. Accidente cerebrovascular trombótico en paciente con COVID-19, reporte de un caso. Revista científica INSPILIP V. 2020;6-12. Disponible en:

https://www.researchgate.net/publication/341165246

31.Higgs A, McGrath A, Goddard C, Suntharaligam G, Gale R, Cook T, et al. Guidelines for the management of tracheal intubation in critically ill adults. British Journal of Anaesthesia. 2018;323-352. Disponible en: https://doi.org/10.1016/j.bja.2017.10.021

32. Monares E, Chavarria U, Sánchez J. Notas del manejo hemodinámico durante la guardia COVID-19. Revista Mexicana de Anestesiología. 2020;43(2):140-144. https://dx.doi.org/10.35366/92873

33.Alhazzani W, Moller M, Arabi Y, Loeb M, Gong M, Fan E. Surviving Sepsis Campaign: guidelines on the management of critically ill adults with Coronavirus Disease 2019 (COVID-19). Intensive Care Med. 2020;46:854-887. https://doi.org/10.1007/s00134-020-06022-5

34.Lamontagne F, Richards-Belle A, Thomas K, et al. Effect of Reduced Exposure to Vasopressors on 90-Day Mortality in Older Critically III Patients With Vasodilatory Hypotension: A Randomized Clinical Trial. JAMA Netwok. 2020;323(10):938-949.

https://doi.org/10.1001/jama.2020.0930

35.Johnson M, Moorhead S, Bulechek H, Meridean M, Swanson E. Vínculos de NOC y NIC a NANDA-I y diagnósticos médicos. Soporte para el razonamiento crítico y la calidad de los cuidados. $3^{a}$ ed. España: Elsevier; 2012.

36.Achury D, Achury L, Rodriguez S, Díaz J. Cuidado de Enfermería en la administración de medicamentos por vía intravenosa en: Achury D, Achury L, Rodríguez S, Díaz J. editores. Fundamentos enfermeros en el proceso de administración de medicamentos. Editorial Javeriana. 2012:257-288. 
37.Loubani 0, Green R. A systematic review of extravasation and local tissue injury from administration of vasopressors through peripheral intravenous catheters and central venous catheters. Journal of Critical Care. 2015:30(3):653.e9-653.e6.53E17.

https://doi.org/10.1016/j.jcrc.2015.01.014

38.Brewster D, Chrimes N, Do T, Fraser K, Groombridge C, Higgs A, et al. Consensus statement: Safe Airway Society principles of airway management and tracheal intubation specific to the COVID- 19 adult patient group. Medical Journal of Australia. 2020;212(10). https://doi.org/10.5694/mja2.50598

39.Driver B, Klein L, Prekker M, Cole J, Satpathy R, Kartha G, et al. Drug Order in Rapid Sequence Intubation. Society for Academic Emergency Medicine. 2019;00:1-8.

https://doi.org/10.1111/acem.13723

40.Farkas J. PlumCrit- Rocketamine vs. Keturonium for Rapid Sequence Intubation. PulmCrit. 2017. Disponible en: https://emcrit.org/pulmcrit/pulmcrit-rocketamine-vs-keturonium-rapid-sequence-intubation/

41.Tafur L, Serna A, Lema E. Fentanilo PK/PD, un medicamento vigente. Rev. Col. Anest. 2010;38(1):68-83. https://doi.org/10.1016/S0120-3347(10)81005-6

42.Organización Panamericana de la Salud. Documento del gobierno POS/HSS/MT/COVID-19/20-0019. Lista de medicamentos esenciales para el manejo de pacientes que ingresan a unidades de cuidados intensivos con sospecha o diagnóstico confirmado de COVID-19. 2020; 3-6. Disponible en: https://iris.paho.org/bitstream/handle/10665.2/52192/OPSHSSMTCOVID- 19200019_spa.pdf?sequence=1\&isAllowed=y

43.Celis E, Birchenall C, De la Cal M, Castorena G, Hernández A, Ceraso D. et al. Guía de práctica clínica basada en la evidencia para el manejo de la sedoanalgesia en el paciente adulto críticamente enfermo. Med Intensiva. 2013;37(8):519-574.

http://dx.doi.org/10.1016/j.medin.2013.04.001

44.Aranda F, Aliste J, Altermatt F, Alvarez J, Bernucci F, Bruhn A, et al. Recomendaciones para el manejo de pacientes con COVID-19 con indicación terapéutica de ventilación mecánica que eventualmente son conectados a máquinas de anestesia. Rev Chil Anest. 2020;49(3) https://doi.org/10.25237/revchilanestv49n03.09

45.Herdman T, Kamitsuru, S. Diagnósticos enfermeros. Definiciones y clasificación 2018-2020. $11^{\text {a }}$ ed. España: Elsevier; 2018.

46.Maluenda F, Aguilera P, Kripper C, Navea O, Basaure C, Saldias F. Secuencia rápida de intubación en el Servicio de Urgencia. Revista Chilena De Medicina Intensiva. 2015. [Consultado el 15 de junio de 2020]; 23-32. Disponible en: https://www.studocu.com/es/document/universidad-de-los-andes-venezuela/fisiologia/otros/secuencia-rapida-de-intubacion-en-el-servicio-de-urgencia/2143892/view

47.López E, Miana M, Molas G. Especialidades clínicas: paciente crítico en Zabalegui A, Lombraña M, Mangues I, Tuneu L, Codina C, Molina J. editores. Administración de medicamentos y cálculos de dosis. Segunda edición. Elsevier España 2014.

48.Achury D, Achury L. Cuidado de Enfermería en la administración de medicamentos especiales en Achury D, Achury L, Rodriguez S, Díaz J. editores. Fundamentos enfermeros en el proceso de administración de medicamentos. Editorial Javeriana. 2012. p. 409-432. 